\section{Compliance to Intraoperative Basic Hygiene and Patient Safety Culture in Mozambique}

\section{Abstract}

Background: Surgical site infections are commonly occurring within healthcare, especially in Africa. Good hygiene is the most effective way in which to reduce and prevent infection. However, compliance is often low or insufficient.

Objective: To assess intraoperative compliance to basic hygiene in the operating theatre and the staffs' views on patient safety and to assess whether adherence to hand hygiene is related to patient safety culture in a developing country.

Methods: The design was a structured observation in order to gathered information on compliance to basic intraoperative hygiene routines in operating theatres in Mozambique. Theatre staff was also asked to complete a survey on patient safety culture.

Results: The study reveals that none of the work elements were performed in complete compliance to WHO's guidelines at all times. The theatre staff's views on patient safety culture showed the highest percentage of positive responses was within "Teamwork Within Hospital Units" and the dimensions with the least positive response was "Nonpunitive Response To Error" and "Staffing". A medium relation was found between compliance to basic hygiene and the results of the patient safety culture survey.

Conclusion: This study shows that compliance to basic hygiene during the intraoperative phase in theatre was insufficient. There was a medium relation between the views of the staff on patient safety and their compliance to basic hand hygiene. This implies that working with the attitudes of the staff concerning patient safety could be one way of improving hygiene compliance which would be expected to reduce the number of surgical site infections.

Keywords: Hygiene; Hand-hygiene; Compliance; Operating theatre; Patient safety culture

\section{Oscarsson $\mathbf{R}$ and Swenne CL \\ Uppsala University, Uppsala, Sweden}

\section{Corresponding Author:}

Christine Leo Swenne

झ christine.leo.swenne@pubcare.uu.se

Uppsala University, Box 564, Uppsala, Sweden.

Tel: + 46184716619

Citation: Oscarsson R, Swenne CL.

Compliance to Intraoperative Basic Hygiene and Patient Safety Culture in Mozambique. J Prev Infect Control. 2016, 2:1.

\section{Introduction}

Millions of surgical procedures are performed worldwide every year. The majority of surgical wounds heal without complications. However, thousands of infections are recorded nationally every year post surgery, making surgical site infections commonly occurring in healthcare, ranging from superficial skin infections to much more severe conditions some in fact life-threatening [1]. A review studying surgical site infections in low- income countries and Africa in particular found that the frequency of surgical site infections were shockingly high in comparison to high-income countries [2].

Patients going into surgery may potentially already have several risk factors for infection [3]. A preventive measure such as good hand hygiene compliance is a widely recognised effective way in which to reduce infections [4] and can therefore improve the patient's outcome after surgery [3]. In an operating theatre much care is taken to maintaining sterility in the operating field but less attention is given to basic hand hygiene. Failing to apply correct hand hygiene before and after patient contact can lead to contamination to non-sterile surfaces or implements that may later cross-infect to another patient [5].

\section{Basic hygiene recommendations according to WHO in order to prevent infection are}

- Correct dress code in accordance with local policies, short 
sleeved scrub suit which are changed daily or more often if needed. No jewelry on underarms or hands, this also includes no wristwatches.

- Hands should be cleaned with water and soap if visibly dirty.

- Hands should always be disinfected before and after all patient contact and before and after all glove use.

- Gloves should be used when there is a risk of contact with blood, non-intact skin, any type of body-fluid, excretion, secretion or mucous membranes. Gloves should be removed before contact with non-contaminated surfaces or objects.

- A gown or an apron should be used in order to protect clothes from being soiled whenever doing activities likely to cause splashes of urine, blood or other body fluids.

Extremely low rates of hand hygiene have been documented in sub- Saharan Africa, hand hygiene being one of the cornerstones in controlling infection [6]. Despite knowledge of the benefits of basic hygiene routines compliance often remains low among health staff [4].

It is the theatre nurse responsibility to uphold a good standard of hygiene in the operating theatre to ensure the surgical procedure is performed in a safe manner, thus preventing and limiting postsurgical infections.

\section{Hygiene routines specific for intraoperative care according to WHOs guidelines for Safe Surgery}

- Clean environment; operating theatres should be situated apart from the rest of the hospital to prevent spread of infection. Walls, floors and all surfaces kept clean with water and an alcohol-based solution.

- Ventilation; a well-functioning and controlled ventilation system reduces airborne contamination and reduces airborne bacteria.

- Pre-surgical skin disinfection; the area planned for surgical incision must be cleaned with a disinfectant approved for presurgical skin preparation in order to significantly reduce the number of microorganisms and colony forming units.

- Sterile dressed staff; surgical attire should consist of masks that cover nose and mouth, hair-covering caps, surgical robes and sterile gloves.

- Guaranteeing the sterility of surgical instruments; all instruments used when entering body tissue must be sterilized, sterile instruments should be cleaned from all living microorganisms or the possibility of there being any remaining microorganisms are equal to or less than one in a million.

- Surgical draping; the area for incision should be surrounded by sterile surgical draping covering the patient completely [7].

Implementing sustainable healthcare improvements is challenging in all sets but in low-income countries examples of enforcing evidence-based practices is limited [8]. Furthermore resources are limited, but surgery can still be performed at an acceptable level of care. It is however challenging, teamwork is of great importance and everyone must learn to work with the equipment that is available to them [9]. To understand why compliance remains low one must understand the staffs' reason to follow or not to follow hygiene guidelines [10]. A study done by Whitby et al. [11], describes an action such as handwashing behavior as complex. Several theories have been developed in order to find a relationship between factors and its effect on health- related behavior. One of these theories is the Theory of planned behavior which describes a person's intention to perform a behavior, in this case hygiene routines is predicted through three different variables [11]. Behavioral beliefs; beliefs on the outcome of the action and the evaluation of the outcomes, normative beliefs; beliefs on the normative expectation from others and the motivation to perform accordingly to them and lastly the control beliefs; which is the beliefs of factors that may help or prevent the performance of the action [12]. These variables are created as a result from our biological characteristics, education, environment and culture and control the outcome of the behavior [11].

The theory of planned behavior can be linked to the patient safety culture, a specific aspect of the general organisational culture shaped by prevailing norms, values and beliefs in the organization [13]. This culture within healthcare is shaped due to the leaders and co-workers attitudes and approach to patient safety. Approaches and attitudes are mirrored in the safety within the ward or healthcare organisation and is often measured through questionnaires or surveys [14]

Surgical site infections can be prevented however, studies show that hand hygiene is not followed optimally in developing countries [15]. Compliance to basic hand hygiene should therefore be prioritised in order to optimize patient safety. Reason for performing these actions is affected by general culture but more specifically in health-care patient safety culture. Knowledge of reasons for basic hygiene guidelines not being performed in developing countries must be studied and understood in order to improve patient safety and reduce infections. The aim of the present study was to assess intraoperative compliance to basic hygiene in the operating theatre and the staffs' views on patient safety and to assess whether adherence to hand hygiene is related to patient safety culture in a developing country.

\section{Materials and Methods}

\section{Study location}

The study took place in a private hospital in Mocambique. The population of the study was all operating theatres staff. A convenience sampling was used where all staff present at the time of the observation was included.

\section{Study design}

The study employed a structured observation approach with a descriptive design.

\section{Study population}

A total of 24 nurses were observed. Fifteen nurses both anesthetic and scrub nurses completed the patient safety culture survey, both male and female, between 26-67 years of age. Surgeons and anaesthesiologist did not partake in the patient safety culture survey. The operating team consisted of 5-10 people depending on the procedure and included; scrub nurses, anesthetic nurses, anesthesiologists and surgeons. Scrub nurses education varied, some had nursing degrees with previous general nursing experience and had later been schooled as scrub nurses either in 
Europe in a one year training program, or in Mozambique where they were trained by scrub nurses with European degrees. Some of the staff didn't have a nursing degree but had been trained to assist and instrument in surgery as scrub nurses. Scrub nurses would also act as assistant scrub nurses when needed.

\section{Data Collection}

\section{Observation protocol}

The definition of hand hygiene according to the World Health Organisation was used for the purpose of this study [16]. The observational protocol lists ten elements within intraoperative surgery based on a protocol used in a previous study [17]. Ten elements important for basic hygiene and operation hygiene are; 1 ) dress code in operating room, 2) insertion of peripheral venous catheter, 3) spinal anesthetics, 4) anaesthesia procedures consisting of ventilation, intubation, drug administration and performance of transesophageal echocardiogram, 5) insertion of urinary catheter, 6) placing the patient in surgical position, 7) intraoperative skin disinfection, 8) surgical draping, 9) intraoperative management of blood sampling, infusion set, swabs and drainage tubing and 10) asepsis during the surgical procedure.

\section{The Survey on patient safety culture}

The hospital survey on patient safety culture was created 2004 by the Agency for Healthcare Research and Quality and since then it has been used internationally [18]. It is proven to be reliable in countries worldwide, in developed as well as developing countries such as Taiwan and Oman [19]. The Portuguese version has also been assessed and tested in order to ensure its validity after translation [20]. The survey consists of 12 dimensions (Table 1).

\section{Procedures for data collection}

The observer was located in the operating theatre dressed according to the local policies dress code for operating staff. All surgical staff was informed of the observer's participation and the aim of the study prior to each surgery. The observation started when the patient entered the theatre and finished when the dressing had been applied to the surgical wound. Notes were kept in an observational log and were noted during and directly after each surgery. Each participant was coded during observations

Table 1 The 12 dimensions in the Patient safety culture survey.

\begin{tabular}{|c|c|}
\hline 1. & Communication Openness ${ }^{2}$ \\
\hline 2. & Feedback and Communication About Error ${ }^{2}$ \\
\hline 3. & Frequency of Event Reporting ${ }^{2}$ \\
\hline 4. & Hospital Handoffs \& Transitions ${ }^{1}$ \\
\hline 5. & Hospital Management Support for Patient Safety ${ }^{1}$ \\
\hline 6. & Nonpunitive Response To Error ${ }^{1}$ \\
\hline 7. & Organisational Learning-Continous improvement ${ }^{1}$ \\
\hline 8. & Overall Perceptions of Safety ${ }^{1}$ \\
\hline 9. & Staffing $^{1}$ \\
\hline 10. & Supervisor/manager expectations \& actions promoting safety ${ }^{1}$ \\
\hline 11. & Teamwork Across Hospital Units ${ }^{1}$ \\
\hline 12. & Teamwork Within Hospital Units ${ }^{1}$ \\
\hline
\end{tabular}

${ }^{1}$ Response alternative from $1=$ Strongly disagree to $5=$ Strongly agree; ${ }^{2}$ Response alternative from $1=$ Never to $5=$ Always in order to keep track of each participant whilst remaining anonymous and in order to later be matched to the patient safety culture survey. During a two week period, a total of 24 surgeries were observed. Two observations were used as pilot observations in order to adjust the observational protocol. All surgeries where the observer was not present from the very beginning or could not stay for the whole time of the procedure were excluded from the study, this excluded three observations. A total of 19 surgeries were therefore used in this study.

During the second week of observation permission was granted by the theatre manager to ask operating staff to fill in the patient safety culture survey. Due to all employed staff being a relatively small group surveys were personally handed out to all daytime staff working that week during calm working hours. Attached to each survey was a short informative letter of the study in Portuguese. Each survey was coded with a matching code as they had been coded in the observations. The surveys were completed both during working hours and in some of the staffs own time. All surveys that had been handed out were collected 2 weeks after they had first been handed out.

\section{Setting in the theatres}

The operating ward had three spacious operating theatres which were cleaned according to a strict daily schedule. All theatres had ventilation with a laminar air flow system suited for performing surgical operations, however due to difficulty in maintenance not all ventilation monitors were functioning so they could be checked that they were in fact upholding operating theatre temperature, humidity and ventilation standards. Each theatre had four doors, one main sliding door leading to the operating ward from which the patient would enter, the second lead into an anteroom with a dedicated scrub area, the third into a middle room with storage space for instruments and the fourth was a back door leading into the sterile. Thus creating a closed circuit where the main door leading out into the operating ward would not have to be used during surgery. One small hand-disinfectant sanitizers was placed in the corner on top of a low cupboard, gloves in one or two sizes were placed on top of the same cupboard and on the anesthetics drug trolley.

Operating staff were dressed according to local dress policy with operating scrubs, shoe covers and scrub caps. Scrub nurses often used hairbands to guarantee hair did not fall out from the scrub cap. Nurses' hands were kept clean from jewelry and nail polish, however surgeons and anesthesiologists often wore watches and or wore nail varnish. Procedures for insertion of a urinary catheter was listed in a protocol which was checked post every surgery. Prior to the patient entering the operating theatre a sterile table with all equipment needed for insertion of the urinary catheter including a long hemostat for skin disinfection was set up by the scrub nurse. The surgeon responsible for placing the urinary catheter, would wash their hands before applying a plastic apron and sterile gloves. The protocol controlled the method of insertion, working as a quality check but also a reminder of how to correctly insert the urinary catheter according to hygiene guidelines.

\section{Data analysis}

Data from the observational protocol was analysed using Statistical Package for Social Sciences (SPSS). When all observation variables, 
in the observation protocol, of an element for everyone in the team were correctly performed, compliance to hand hygiene and intraoperative hygiene guidelines was rated as $100 \%$ complete, $99-75 \%$ fulfilment was rated as partial and $<75 \%$ was rated as insufficient compliance [17]. The performance of the whole team was compiled to an overall score. When contamination occurred for the first time in a team compliance with guidelines was rated as partial (compliance) even if the next caregiver fulfilled the routine for the procedure.

The answers to each question in the patient safety culture survey were compiled in their dimension and its amount calculated. The dimensions were answered on a scale of 1-5, ranging from strongly disagreeing with a statement to strongly agreeing, or never to always. The percentage and quota was calculated according to the instructions as written in the handbook [14]. The results from the observation protocol and the patient safety culture survey analysis were then compared to see if there was a relation between views on patient safety and the compliance of the staff to basic hygiene. This was done by using Spearman correlation. The results were then interpreted according to Cohen's guidelines on a scale ranging from a small to large correlation [21].

\section{Ethical considerations}

The study followed the Helsinki declaration [22]. The study can hopefully be useful in developing safer patient care, however being observed can be perceived as being judged or scrutinized which could give the participants a negative view of the study. The information of the study was therefore pointed and emphasized that the study aims to benefit the patients and not to judge the staff of their actions. When operating staff did not agree to participate in the study, his or her wishes were respected.

\section{Results}

Nineteen surgical procedures where observed consisting of cesareans, hysterectomies, amputations, cysts, tumors and fistula operations. The time of surgery varied from 20 minutes to five hours, with five to ten people in the operating theatre depending on the procedure.

\section{Observed intraoperative compliance}

None of the work elements were performed in accordance to the hygiene guidelines according to WHO at all times(Table 2). Only three out of the nineteen observations had complete compliance to the dress code in the operating theatre, this due to watches, rings and nail polish often being used. Surgical masks were sometimes worn covering only the mouth leaving the nose exposed.

Insertion of peripheral venous catheter, spinal anaesthesia and anaesthesia procedures consisting of ventilation, intubation and drug administration were performed in partial and insufficient compliance. When inserting a urinary catheter compliance was complete in 8 out of 11 observations. Intraoperative skin disinfection was performed in complete compliance in 14 out of 19 observed surgeries, when the element was not performed in compliance it was due to contamination. Surgical draping was also partial in 5 observations and complete in 14 observations, contamination did not occur in the same observations as the skin disinfection. Management of blood sampling, infusion set, swabs and drainage tubing was never performed in complete compliance. Asepsis during the surgical procedure was complete in 12 observations and partial in 7.

\section{Operating theatre staff's views on patient safety culture}

Strengths in patient safety culture could be seen in the dimension "Teamwork within hospital units" with the most positive responses, this dimension included questions regarding weather the staff supported and respected one another and if the staff worked together as a team when a lot of work needed to be done quickly. "Organisational learning- continous improvement" also received a high positive response, where the staff was asked if they felt they were actively trying to improve patient safety by making positive changes after mistakes were made and evaluating the changes of their effectiveness. "Frequency of event reporting" received $74 \%$ positive response, including questions such as how often mistakes that could potentially harm a patient are reported. The weakest dimensions with the least positive responses was "Nonpunitive response to error" with $17 \%$, suggesting staff often felt mistakes were held against them and that they worried mistakes they made were kept in their personnel file. The second dimension with the least positive responses was "Staffing" meaning the staff often felt they did not have enough staff to handle their workload, and that they often had to work harder and longer than was best for the patient (Figure 1).

\section{Relation between compliance to basic hand hygiene and the results of the patient safety culture survey}

The relation between the responses to the dimensions combined and the personnel's compliance to basic hygiene was a negative medium correlation between the two variables $(r=-0.35, n=12$ $p=0.017$ ) (Table 3). Suggesting that there is a relation between the two, the higher the personnel rated patient safety the better their compliance to hygiene routines. When comparing the dimensions individually to the operating theatre personnel's compliance to basic hygiene routines the strongest relation was seen between the 5th dimension in the patient safety hospital survey, "Hospital management support for patient safety" Spearman's rho=-0.7 $(r=0 .-70, n=12, p=0.008)$ This implies that the higher the staff rated the hospital management support for patient safety the better their compliance was.

A large and therefore strong relation was also found between "Communication openness" $(r=0.56, n=12, p=0,076)$, this relationship was a positive relation meaning that the higher the personnel rated communication openness the lower their compliance was (Table 2).

\section{Discussion}

None of the work elements in the current study were performed in complete compliance to WHO's guidelines at all times, proving that compliance to basic hygiene often falls short of acceptable standards [15].The theatre staffs views on Patient safety culture 
showed the highest percentage of positive responses was the dimensions "Teamwork within hospital units" and "Organisational learning-continous improvement". The dimensions with the least positive response were "Nonpunitive response to error" and "Staffing". When comparing compliance to basic hygiene and the results of the patient safety culture survey a medium relation was found, where the staff who gave the most positive response to the survey also complied better to the WHO's hygiene guidelines. In this study compliance to basic hygiene guidelines was often insufficient, insertion of peripheral venous catheter, spinal anesthetics and anesthesia procedure where never performed in complete compliance in the observed procedures. Due to lack of available hand-disinfectant sanitizers in the operating theatre, staff could not sanitize hands prior or after completing each procedure, resulting in partial or insufficient compliance. Oludra et al. [9], describe how performing surgery in countries where resources are limited as challenging, the staff must make use of the equipment that is available to them. Theatre staff often wore

\section{PATIENT SAFETY CULTURE DIMENSIONS}

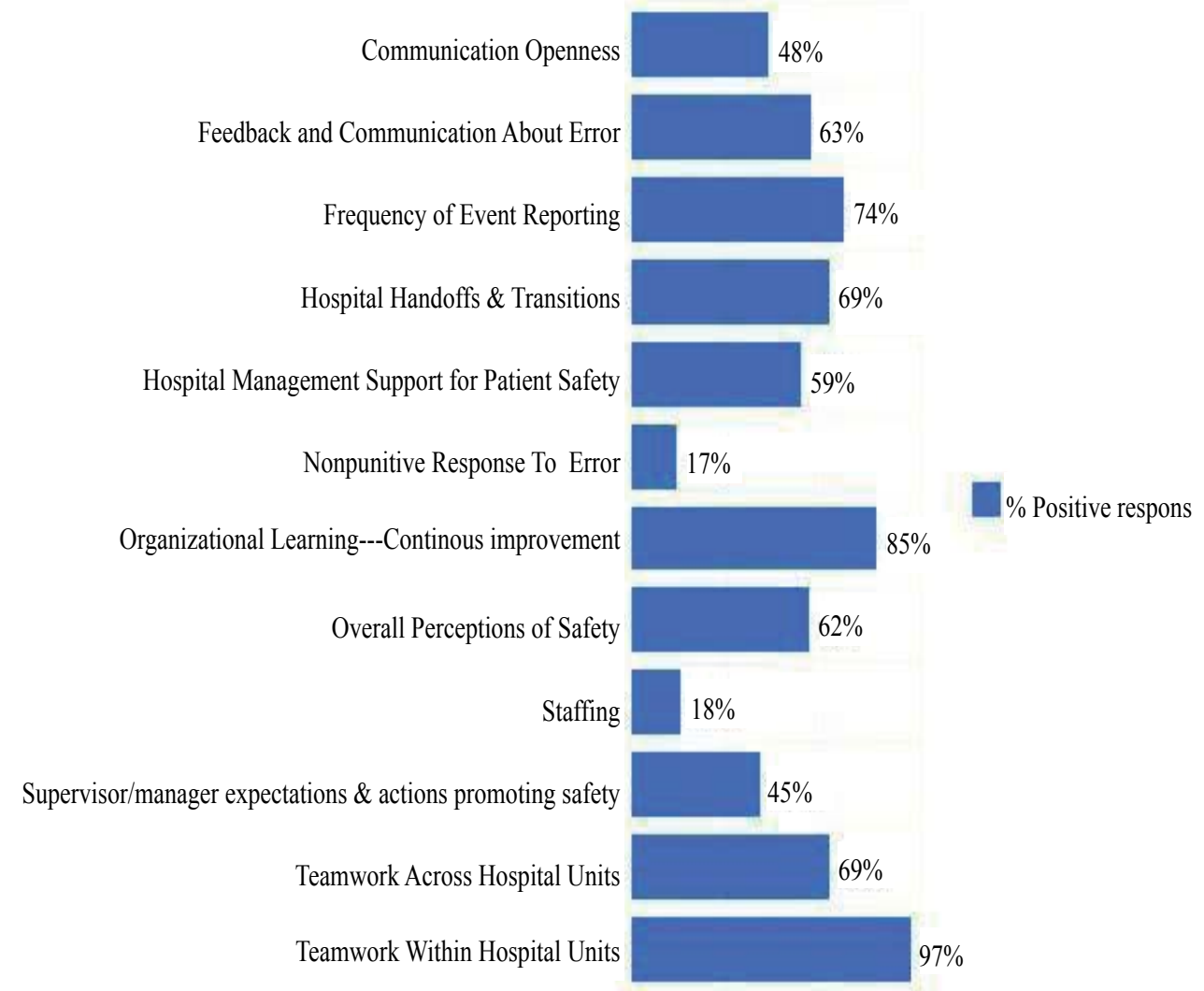

Figure 1 Operating theatre personnel's views on patient safety culture, $n=15$.

Table 2 Observed intraoperative compliance, $\mathrm{n}=19$.

\section{Work Elements}

Dress code in Operating ward, number of observations, $(n=19)$

Insertion of peripheral venous catheter $\mathrm{n}=11$

Spinal $n=7$

Anaesthesia $\mathrm{n}=15$

Insertion of urinary catheter $\mathrm{n}=11$

Placing patiens in surgical position $n=14$

Intraoperative skin disinfection $\mathrm{n}=19$

Surgical draping $\mathrm{n}=19$

Intraoperative management of blood sampling, infusion set, swabs and

drainage tubing $n=10$

Asepsis during the surgical procedure $n=19$
Complete Complience Partial compliance Insufficient Compliance

$(100 \%)$

3

0

0

0

8

5

14

14

$(60-99 \%)$ $(<60 \%)$

7

9

92

$7 \quad 0$

\begin{tabular}{l|l|l}
\hline 14 & 1
\end{tabular}

3

8

5

30

$8 \quad 1$

5

5 
Table 3 The relation between compliance to basic hand hygiene and views on patient safety culture.

\begin{tabular}{|c|c|c|c|}
\hline Dimensions & Small ( $r=0.10$ to 0.29$)$ & Medium ( $r=0.30$ to 0.49 ) & Large $(r=0.50$ to 1.0$)$ \\
\hline Communication openness & - & - & $r=0.56$ \\
\hline Feedback and communication about error & $r=0.22$ & - & - \\
\hline Frequency of event reporting & $r=0.13$ & - & - \\
\hline Hospital handoffs and transitions & $r=0.06$ & - & - \\
\hline Hospital management support for patient safety & - & - & $r=0.70$ \\
\hline Nonpunitive response to error & $r=0.1$ & - & - \\
\hline Organisational learning - continuous improvement & $r=0.04$ & - & - \\
\hline Overall perceptions of safety & - & $r=0.47$ & - \\
\hline Staffing & $r=0.03$ & - & - \\
\hline Supervisor/Manager expectations \& actions promoting safety & - & $r=0.33$ & - \\
\hline Teamwork across Hospital units & $r=0.13$ & - & - \\
\hline Teamwork within Hospital units & $r=0.26$ & - & - \\
\hline
\end{tabular}

gloves when placing the patient in surgical position in order to protect themselves and the patient from contamination, this is an example of using the available equipment at hand when handdisinfectant sanitizers is not available. Even though by doing so, glove use was in fact improper, since gloves should only be used when there is a risk of contact with blood, non-intact tissue or other body fluids [7].

Intraoperative skin disinfection, surgical draping and asepsis during procedure were however performed in complete compliance to the guidelines in several observations. Theatre nurses were skilled in maintaining a sterile environment, thus supporting the theory that much care is taken to maintain asepsis during procedures, but less to basic hand hygiene [5].

Compliance to hygiene when inserting a urinary catheter was partial in 3 and complete in 8 of the observations, reason for compliance being complete may have been a local protocol being followed, thus checking hygiene steps were followed as intended. Urinary tract infections caused by catheters is the most commonly acquired infections within hospitals. The most important step in preventing infections is correct insertion of the urinary catheter, wards or hospitals that introduce strict guidelines on prevention of catheter-associated urinary tract infection have been able to decrease the frequency urinary infections [23].

"Teamwork within hospital units" received the highest positive response out of all the dimensions in the hospital patient safety culture survey similar to the results of Nordin et al. [13] study performed in Sweden and Chen et al. [24] in Taiwan. Wellfunctioning team work contributes to safer patient safety on the other hand "Nonpunitive response to error" and "Staffing" had a very low positive response percentage also similar to studies in Taiwan [13] meaning majority of the operating staff found that they were not enough staff to handle the workload at hand. "Frequency of event reporting" received $74 \%$ positive response in this study, reporting of events is an important factor in order to improve healthcare and prevent harm [25]. However the negative response "Nonpunitive response to error" meant staff often feel like their mistakes are held against them and that staff worry mistakes they make are kept in their personnel file. If staff felt their mistakes are held against them staff may refrain from reporting an error. When observing the staff the author could see a hierarchy within the organisation and a punishment culture. If staff feels they are not respected as equals they may feel that they are judged by the higher organisation when mistakes are made, making them feel as though problems are held against them (Table 3).

The personals approach and attitude towards patient safety is shaped due to norms, values and beliefs of the organisation and according to the theory of planned behavior predicts the staffs intention to perform an intention such as handwashing $[11,14]$. When comparing the operating staffs' compliance to basic hygiene and the staffs' views on patient safety we could find a medium relation between their responses and how well the staff complied with hygiene routines. The more positive view of patient safety the better their compliance was, which could mean the better the staff felt about hospital management support for patient safety and their overall perception of patient safety the better they themselves performed in accordance with the guidelines as is expected of them.

Thousands of surgical site infections are recorded every year [1]. Causing prolonged hospital stays, patient suffering and increased hospital costs [26]. Finding factors that could prevent surgical site infections is therefore of importance for the individual patient, healthcare and society as a whole. The current study also suggests that intraoperative hygiene is often flawed. Further studies observing why hygiene guidelines are not followed as intended would strengthen the evidence. The context is the hospitals possibility to put the evidence into action.

The results of the current study was presented to the staff responsible for infection- control, knowledge of the results from the current study could lead to knowledge and an understanding of flawed areas within the organisation that may be in need of change and improvement. The technique in which the information and knowledge are then implemented is the facilitation [14]. However due to hierarchies within the organisation at the hospital of the study implementation of improvements was difficult, staff higher in hierarchy were often not receptive to changes, when the suggestion of improvement came from a staff member lower in rank than themselves. Change would have to be put into action from the top management in order for all staff to respect and act accordingly to the changes. Alternately Gagliardi et al. [27] suggest a knowledge translation within the same profession, where one person educates or mentors another as an efficient way of implementing change. Educating staff within each profession in order to implement change could therefore 
be better suited within organisations with hierarchical cultures. Hierarchy within healthcare is a common recurring problem within healthcare [28].

Apart from the leaders and organisation responsibility of upholding a safe standard within healthcare, nurses ethics code state that nurses have a responsibility of working and acting alongside guidelines and up-to-date research within their profession [29]. Theatre nurses are responsible for hygiene and asepsis during surgery but also for providing the best possible care for the patient, following guidelines on basic hygiene in order to prevent surgical site infections is therefore of utmost importance. In the current study views on patient culture did have a relation to how the staff complied with basic hygiene guidelines. Knowledge of reasons for staff not to follow guidelines must always be analysed in order to develop healthcare[4] and perform safer surgery in order to stop the chain reaction of patient surgical site infections, prolonged hospital stays, patient suffering and increased hospital costs.

\section{Limitations}

The official language of Mozambique is Portuguese, not speaking the official language may have limited the study since a better understanding of culture and understanding of observations could have been made. The fist author has lived in Africa, but previous knowledge may also affect or angel the study without the author's purpose or knowledge. All observations were done by a single observer, to improve reliability two observers would have been preferable in order test the interrater reliability, where the observations of both observers would have been compared in order to see that they had both came to the same conclusion.

The Hospital patient safety culture survey has been tested and proven to be both a reliable and valid instrument for measuring patient safety culture [30]. The Portuguese translation of the survey has also been tested and proven reliable [20]. Two pilot observations where done in order to confirm the observational protocol was a valid instrument to measure the operating staff's compliance. After the pilot studies, spinal aesthetics was added to the protocols work elements, since it was a common recurring procedure. The patient safety culture survey validity has been tested in previous studies [30]. Both instruments for measurement have previously used and proven internally valid.

The study was conducted in an operating ward with 22 nurses working at the time of the study and the response rate to the patient safety culture survey was $75 \%$. Surgeons and anaesthesiologist did not partake in the patient safety culture survey and the hospital was small and private in Mozambique. The results of the study can therefore not be generalized to a whole culture or all developing countries. Similar studies would need to be conducted in developing countries in order to generalize the results to a country or culture.

\section{Clinical implications}

This study performed in Mozambique shows that compliance to basic hygiene during the intraoperative phase in theatre was insufficient. There was a medium strong relation between the views of the staff on patient safety and their compliance to basic hygiene. This implies that working with the attitudes of the staff concerning patient safety could be one way of improving hygiene compliance which would be expected to reduce the number of surgical site infections.

\section{Acknowledgement}

The author is grateful to all clinical staff for their cooperation with participation in this study. 


\section{References}

1 Korol E, Johnston K, Waser N, Sifakis F, Jafri HS, et al. (2013) A systematic review of risk factors associated with surgical site infections among surgical patients. PLoS One 8: e83743.

2 Aiken AM, Wanyoro AK, Mwangi J, Mulingwa P, Wanjohi J, et al. (2013) Evaluation of surveillance for surgical site infections in Thika Hospital, Kenya. J Hosp Infect 83: 140-145.

3 Fry DE (2013) The prevention of surgical site infection in elective colon surgery. Scientifica (Cairo) 2013: 896297.

4 Lee SS, Park SJ, Chung MJ, Lee JH, Kang HJ, et al. (2014) Improved Hand Hygiene Compliance is Associated with the Change of Perception toward Hand Hygiene among Medical Personnel. Infect Chemother 46: 165-171.

5 Krediet AC, Kalkman CJ, Bonten MJ, Gigengack AC, Barach P (2011) Hand-hygiene practices in the operating theatre: an observational study. Br J Anaesth 107: 553-558.

6 Schmitz K, Kempker RR, Tenna A, Stenehjem E, Abebe E, et al. (2014) Effectiveness of a multimodal hand hygiene campaign and obstacles to success in Addis Ababa, Ethiopia. Antimicrob Resist Infect Control 3: 8

7 World Health Organization (2009) WHO Guidelines for Safe Surgery 2009

8 Aiken AM, Wanyoro AK, Mwangi J, Juma F, Mugoya IK, et al. (2013) Changing Use of Surgical Antibiotic Prophylaxis in Thika Hospital, Kenya: A Quality Improvement Intervention with an Interrupted Time Series Design. PLoS One. 8: e78942.

9 Oludara MA, Nwiloh J, Fabamwo A, Adebola P (2014) Commencing open heart surgery in resource limited countries: lessons from the LASUTH experience. Pan Afr Med J 19: 105.

10 Chavali S, Menon V, Shukla U (2014) Hand hygiene compliance among healthcare workers in an accredited tertiary care hospital. Indian J Crit Care Med 18: 689-693.

11 Whitby M, McLaws ML, Ross MW (2006) Why healthcare workers don't wash their hands: a behavioral explanation. Infect Control Hosp Epidemiol 27: 484-492.

12 Javadi M, Kadkhodaee M, Yaghoubi M, Maroufi M, Shams A (2013) Applying theory of planned behavior in predicting of patient safety behaviors of nurses. Mater Sociomed 25: 52-55.

13 Nordin A, Wilde-Larsson B, Nordström G, Theander K (2013) Swedish Hospital Survey on Patient Safety Culture- Psychometric properties and health care staff's perception. Open Journal of Nursing 3: 41-50.

14 National Board of Health and Welfare (2009) Att mäta patientsäkerhetskulturen.

15 Braimoh OB, Udeabor SE (2013) Hand hygiene practices among community Health Officers in Rivers State, Nigeria. Afr Health Sci 13: 507-511.
16 Magiorakos AP, Leens E, Drouvot V, May-Michelangeli L, Reichardt C, et al. (2010) Pathways to clean hands: highlights of successful hand hygiene implementation strategies in Europe. Euro Surveill 15.

17 Swenne CL, Alexandrén K (2012) Surgical team members' compliance with and knowledge of basic hand hygiene guidelines and intraoperative hygiene. Journal of Infection Prevention 13: 114-119.

18 Agency for healthcare research and quality (2015) Hospital Survey on Patient Safety Culture.

19 Al-Mandhari A, Al-Zakwani I, Al-Kindi M, Tawilah J, Dorvlo AS, et al. (2014) Patient safety culture assessment in oman. Oman Med J 29: 264-270.

20 Reis CT, Laguardia J, Martins M (2012) Translation and cross-cultural adaptation of the Brazilian version of the Hospital Survey on Patient Safety Culture: initial stage. Cad Saude Publica 28: 2199-2210.

21 Pallant J (2005) SPSS survival manual: a step by step guide to data analysis using SPSS for Windows (2 ed.) Buckingham: Open University Press.

22 World Medical Association (2013) World Medical Association Declaration of Helsinki: ethical principles for medical research involving human subjects. JAMA 310: 2191-2194.

23 Jain M, Dogra V, Mishra B, Thakur A, Loomba PS (2015) Knowledge and attitude of doctors and nurses regarding indication for catheterization and prevention of catheter-associated urinary tract infection in a tertiary care hospital. Indian J Crit Care Med 19: 76-81.

24 Chen C, Li HH (2010) Measuring patient safety culture in Taiwan using the Hospital Survey on Patient Safety Culture (HSOPSC). BMC Health Serv Res 10: 152

25 Nabhan M, Elraiyah T, Brown DR, Dilling J, LeBlanc A, et al. (2012) What is preventable harm in healthcare? A systematic review of definitions. BMC Health Serv Res 12: 128.

26 Brisibe S, Ordinioha B, Gbeneolol PK (2014) Knowledge, attitude, and infection control practices of two tertiary hospitals in Port-Harcourt, Nigeria. Niger J Clin Pract 17: 691-695.

27 Gagliardi AR, Webster F, Straus SE (2015) Designing a knowledge translation mentorship program to support the implementation of evidence-based innovations. BMC Health Serv Res 15: 198.

28 Shah N, Castro-Sánchez E, Charani E, Drumright LN, Holmes AH (2015) Towards changing healthcare workers' behaviour: a qualitative study exploring non-compliance through appraisals of infection prevention and control practices. J Hosp Infect 90: 126-134.

29 The Swedish Society of Nursing (2014) ICN;s Code of Ethics for Nurses.

30 Farup PG (2015) Are measurements of patient safety culture and adverse events valid and reliable? Results from a cross sectional study. BMC Health Serv Res 15: 18. 